Pacific Journal of Mathematics

OPERATORS OF MEROMORPHIC TYPE WITH MULTIPLE 


\section{OPERATORS OF MEROMORPHIC TYPE WITH MULTIPLE POLES OF THE RESOLVENT}

JoHn DeRr AND ANgus E. TAYLOR

1. Introduction. Let $X$ be a complex Banach space and let $A$ be a bounded linear operator on $X$ such that the spectrum $\sigma(A)$ is a denumerable set of points with $\lambda=0$ as the only point of accumulation. Suppose, also, that each nonzero point of $\sigma(A)$ is a pole of the resolvent $R_{\lambda}(A)$. Then we shall call $A$ an operator of meromorphic type.

Let $\lambda_{1}, \lambda_{2}, \cdots$ be an enumeration of the distinct nonzero points of $\sigma(A)$, and let $E_{n}$ be the residue of $R_{\lambda}(A)$ at $\lambda_{n}$. Then $E_{n}^{2}=E_{n}, E_{n} \neq 0, E_{n} \neq I$, and $E_{n} E_{m}=0$ if $m \neq n$. Moreover, $E_{n}$ commutes with $A$. If the order of the pole at $\lambda_{n}$ is $q_{n}$, then

$$
\left(A-\lambda_{n}\right)^{q_{n}} E_{n}=0, \quad\left(A-\lambda_{n}\right)^{q_{n}-1} E_{n} \neq 0 .
$$

When $q_{n}>1$, the singular part of the Laurent expansion of $R_{\lambda}(A)$ in the neighborhood of $\lambda_{n}$ is

$$
S_{n}(\lambda)=\frac{E_{n}}{\lambda-\lambda_{n}}+\sum_{k=2}^{q_{n}} \frac{F_{n}^{k-1}}{\left(\lambda-\lambda_{n}\right)^{k}},
$$

where

$$
F_{n}=\left(A-\lambda_{n}\right) E_{n}
$$

In this case

$$
F_{n}^{q n^{-1}} \neq 0, \quad F_{n}^{q n}=0
$$

If $q_{n}=1$, we have

$$
S_{n}(\lambda)=\frac{E_{n}}{\lambda-\lambda_{n}}, \quad F_{n}=0
$$

Observe that $E_{n} F_{n}=F_{n} E_{n}=F_{n}$. For the relevant facts about the coefficients in the Laurent expansion of $R_{\lambda}(A)$ in the neighborhood of an isolated singularity, see Taylor [5]. (Numbers in square brackets refer to the works cited at the end of the paper.)

Throughout this paper we shall be concerned with series expansions of operator-valued functions of $\lambda$. All convergence questions are examined from the point of view of the uniform topology in the space of bounded linear operators on $X$. (We shall denote this space of operators by $[X]$.) Thus, if $T_{n}$ and $T$ are elements of $[X], T_{n} \rightarrow T$ means $\left\|T_{n}-T\right\| \rightarrow 0$.

Received February 26, 1961. 
Because $S_{n}(\lambda)$ is regular when $|\lambda|>\left|\lambda_{n}\right|$, it has an expansion in powers of $1 / \lambda$, convergent when $|\lambda|>\left|\lambda_{n}\right|$. If $p$ is a positive integer, we denote by $P_{n}^{(p)}(\lambda)$ the sum of the terms of degree $\leqq p$ in $1 / \lambda$ in this expansion of $S_{n}(\lambda)$. An explicit formula will be given presently.

It turns out that a sequence $\left\{p_{n}\right\}$ can be chosen in such a way that the series

$$
\sum_{n=1}^{\infty}\left[S_{n}(\lambda)-P_{n}^{\left(p_{n}\right)}(\lambda)\right]
$$

converges when $\lambda$ is not in $\sigma(A)$, and such that, moreover, the difference

$$
R_{\lambda}(A)-\sum_{n=1}^{\infty}\left[S_{n}(\lambda)-P_{n}^{\left(p_{n}\right)}(\lambda)\right]
$$

has removable singularities at the points $\lambda_{1}, \lambda_{2}, \cdots$ and coincides in the resolvent set $\rho(A)$ with a function given by a series

$$
\sum_{n=0}^{\infty} \frac{Q_{n}}{\lambda^{n}}
$$

which converges for every nonzero $\lambda$. Here $Q_{0}, Q_{1}, \cdots$ are bounded linear operators. The sequence $\left\{p_{n}\right\}$ is not unique. It may be chosen along with the imposition of rather strong conditions on the mode of convergence of the series (5). In a paper by one of us (Taylor [6]) a study was made of the situation when all the poles are of the first order and a uniform convergence condition was imposed on (5). Subsequently, in a paper delivered at the Jerusalem Symposium on Linear Spaces, in July 1960, Taylor discussed the situation when an absolute and uniform convergence condition is placed on the series

$$
\sum_{n}\left\|S_{n}(\lambda)-P_{n}^{\left(p_{n}\right)}(\lambda)\right\| \text {. }
$$

Most of the results reported on by Taylor were for the case of simple poles. The theory for the multiple-pole case was begun by Derr in his doctoral dissertation [2]. Some contributions to the multiple-pole theory, especially where the condition of absolute convergence is imposed, were made by Taylor. In this paper we concentrate on the discussion of the multiple-pole case.

In Taylor [7] the following theorem was proved. We repeat the statement here for convenience.

THEOREm 1. If $A$ is of meromorphic type and if the foregoing notation is established, then there exists a sequence $\left\{p_{n}\right\}$ of positive integers such that for each $\delta>0$, if $m$ is so large that $\left|\lambda_{k}\right|<\delta$ when $k \geqq m$, then the series

$$
\sum_{n=m}^{\infty}\left\|S_{n}(\lambda)-P_{n}^{\left(p_{n}\right)}(\lambda)\right\|
$$


converges uniformly in the part of the plane for which $|\lambda| \geqq \delta$. Moreover, there exists a sequence $\left\{Q_{n}\right\}$ of elements of $[X]$ such that the series (7) converges if $\lambda \neq 0$, and for each $\lambda$ in $\rho(A)$ the resolvent of $A$ has the expansion

$$
R_{\lambda}(A)=\sum_{n=1}^{\infty}\left[S_{n}(\lambda)-P_{n}^{(p} n^{\prime}(\lambda)\right]+\sum_{n=0}^{\infty} \lambda^{-n} Q_{n} .
$$

On the basis of this theorem we make the following definitions: A sequence $\left\{p_{n}\right\}$ of the sort specified in Theorem 1 will be called an absolute index sequence for $A$ relative to $\left\{\lambda_{n}\right\}$. If $\left\{p_{n}\right\}$ is an absolute index sequence such that $p_{n}$ has the same value $p$ for every $n$, we shall say that $A$ admits the absolute index $p$. We do not need to add the phrase "relative to $\left\{\lambda_{n}\right\}$ " in this case, because the terms in the series (9) are nonnegative; on this account, if the series converges uniformly in the manner described, it remains thus uniformly convergent after an arbitrary rearrangement of the order of the terms. If $A$ admits the absolute index $p$, but no smaller absolute index, we say that $A$ has minimal absolute index $p$. The minimal absolute index depends only on $A$, not on any particular order of listing of the poles of $R_{\lambda}(A)$.

Now, it may happen that a sequence $\left\{p_{n}\right\}$ of positive integers has the property that for each $\delta>0$, if $m$ is chosen so large that $\left|\lambda_{k}\right|<\delta$ when $k \geqq m$, then the series

$$
\sum_{k=m}^{\infty}\left[S_{n}(\lambda)-P_{n}^{\left(p_{n}\right)}(\lambda)\right]
$$

converges uniformly in the part of the plane for which $|\lambda| \geqq \delta$. Observe carefully the difference between (9) and (11). The series (9) is numerical, whereas (11) is a series whose terms are members of $[X]$. With this modified condition on the sequence $\left\{p_{n}\right\}$, it will be true that the operator function $F(\lambda)$ defined by

$$
F(\lambda)=\sum_{n=1}^{\infty}\left[S_{n}(\lambda)-P_{n}^{\left(p_{n}\right)}(\lambda)\right]
$$

is analytic on $\rho(A)$, regular at $\lambda=\infty$, and has a pole at each of the points $\lambda_{1}, \lambda_{2}, \cdots$, the singular part of the Laurent expansion in the neighborhood of $\lambda_{n}$ being $S_{n}(\lambda)$. Therefore, under these conditions, $R_{\lambda}(A)-F(\lambda)$ will have removable singularities at the points $\lambda_{1}, \lambda_{2} \cdots$, and will be regular at $\infty$. Accordingly, we shall have a representation of the form (10), where the series involving the $Q_{n}$ 's converges when $\lambda \neq 0$.

A sequence $\left\{p_{n}\right\}$ for which we have uniform convergence as specified in connection with the series (11) will be called a uniform index sequence for $A$ relative to $\left\{\lambda_{n}\right\}$. If $p_{n}=p$ for every $n$, we call $p$ a uniform index for $A$ relative to $\left\{\lambda_{n}\right\}$. We can also define a minimal uniform 
index for $A$ relative to $\left\{\lambda_{n}\right\}$, provided there exists at least one such uniform index. It is to be emphasized that the notion of a uniform index for $A$ must be defined in relation to a specified way of enumerating the poles of $R_{\lambda}(A)$. This is because there is no guarantee that uniform convergence of the series (11) is preserved when the order of the term is rearranged. In fact, an example is known (Berkson [1]) in which $A$ has the uniform index 1 relative to a certain enumeration $\left\{\lambda_{n}\right\}$, but the series (11) can be rearranged in such a way as to be divergent at all points of $\rho(A)$.

In Theorem $10(\S 5)$ we give a necessary and sufficient condition for $A$ to have minimal uniform index $p$ relative to $\left\{\lambda_{n}\right\}$. The case $p=1$ is handled in Theorem $5(\S 3)$. The condition is stated entirely in terms of $\lambda_{n}, E_{n}$, and $F_{n}$. A corresponding necessary and sufficient condition for $A$ to have minimal absolute index $p$ is given in Theorem 13 (§6).

These results are of decisive importance for the theory of operators of meromorphic type. With the criteria of Theorems 10 and 13 it is much easier to test for uniform and absolute indices than would be possible by direct reliance on the definitions. The construction of examples is enormously facilitated.

Another important part of the paper is in $\S 4$, where we discuss the decomposition $A=B+C$ for an operator $A$ of meromorphic type and minimal uniform index 1 relative to $\left\{\lambda_{n}\right\}$. In this decomposition, $B$ is of the same general character as $A$, with $\sigma(A)=\sigma(B)$, and $B$ is canonical in a well-defined sense. Moreover, $B C=C B=0$ and $C$ is quasinilpotent. This decomposition is established in Theorem 8 , and its uniqueness is described in Theorem 9.

2. Some preliminary results. In this section we assemble some results and formulas for later use.

LEMma 1. Suppose that $E, F, \in[X]$ and that

$$
E^{2}=E, \quad E F=F E=F, \quad E \neq 0 .
$$

Suppose that there is a positive integer $q$ such that

$$
F^{q-1} \neq 0, \quad F^{q}=0 .
$$

Then, if $\alpha \neq 0$ and $B=\alpha E+F$, the resolvent of $B$ is

$$
R_{\lambda}(B)=\frac{I-E}{\lambda}+\frac{E}{\lambda-\alpha}+\frac{F}{(\lambda-\alpha)^{2}}+\cdots+\frac{F^{q-1}}{(\lambda-\alpha)^{q}}
$$

(the terms involving $F$ occurring only if $q>1$ ). The spectrum of $B$ consists of 0 and $\alpha$ if $E \neq I$ and of $\alpha$ alone if $E=I$. 
Proof. Let $C$ denote the operator in the right member of formula (15), where $\lambda$ is fixed, with $\lambda \neq 0, \lambda \neq \alpha$. It is an easy matter to verify that $C(\lambda-B)=(\lambda-3) C=I$, whence it follows that $\lambda \in \rho(B)$ and $R_{\lambda}(B)=C$ when $\lambda$ is thus restricted. We leave the calculations to the reader. Now, $\alpha$ is an eigenvalue of $B$. For, if $q>1$, choose $x$ so that $y=F^{q-1} x \neq 0$; this is possible, by (14). Then $E y=y$ because $E F=F$; hence $B y=(\alpha E+F) F^{q-1} x=\alpha y$, because $F^{q}=0$. If $q=1$, choose $x$ so that $y=E x \neq 0$. We can do this, because $E \neq 0$. Then, since $F=0$ in this case, $B y=\alpha E y=\alpha y$. Hence it is certain that $\alpha \in \sigma(B)$, regardless of the value of $q$.

If $I-E \neq 0$, and if $y=x-E x \neq 0$, we see that $B y=0$, because $B=B E$. Hence $0 \in \sigma(B)$ in this case. On the other hand, if $E=I$, a direct calculation shows that $0-B$ has the inverse

$$
\frac{I}{-\alpha}+\frac{F}{(-\alpha)^{2}}+\cdots+\frac{F^{q-1}}{(-\alpha)^{q}}
$$

so that $0 \in \rho(B)$ in this case. This completes the proof of Lemma 1 .

It now follows from general spectral theory that the spectral radius of $\alpha E+F$ is $|\alpha|$, and that $R_{\lambda}(B)$ is given by the series

$$
R_{\lambda}(B)=\sum_{k=0}^{\infty} \frac{(\alpha E+F)^{k}}{\lambda^{k+1}}
$$

when $|\lambda|>|\alpha|$. From (15) we then see that

$$
\frac{E}{\lambda-\alpha}+\sum_{j=1}^{4-1} \frac{F^{j}}{(\lambda-\alpha)^{j+1}}=\frac{E}{\lambda}+\sum_{k=1}^{\infty} \frac{(\alpha E+F)^{k}}{\lambda^{k+1}},
$$

provided that $|\lambda|>|\alpha|$.

The foregoing considerations can be applied to obtain a formula for $P_{n}^{(p)}(\lambda)$, which is, by definition, the sum of the terms of degree $\leqq p$ in $1 / \lambda$ in the expansion of $S_{n}(\lambda)$ in powers of $1 / \lambda$. By comparing (1) and (17) we see that

$$
S_{n}(\lambda)=\frac{E_{n}}{\lambda}+\sum_{k=1}^{\infty} \frac{\left(\lambda_{n} E_{n}+F_{n}\right)^{k}}{\lambda^{k+1}}
$$

if $|\lambda|>\left|\lambda_{n}\right|$. Therefore

$$
\begin{gathered}
P_{n}^{(1)}(\lambda)=\frac{E_{n}}{\lambda}, \\
P_{n}^{(p)}(\lambda)=\frac{E_{n}}{\lambda}+\sum_{k=2}^{p} \frac{\left(\lambda_{n} E_{n}+F_{n}\right)^{k-1}}{\lambda^{k}} \text { if } p \geqq 2 .
\end{gathered}
$$

The formula (18) could also be obtained from (1) by direct use of binomial 
series. This was done, in the first instance, by Derr in his thesis (Derr [2]). The present method, using spectral theory, is rather elegant.

Now that we have the formula (18), it is easy to calculate the formulas for the operator coefficients $Q_{0}, Q_{1}, \ldots$ occurring in (10). For this calculation it is sufficient to assume that $\left\{p_{n}\right\}$ is a uniform index sequence for $A$ relative to $\left\{\lambda_{n}\right\}$. (An absolute index sequence is also a uniform index sequence of course.) From general spectral theory it is known that

$$
\frac{1}{2 \pi i} \oint \lambda^{j} R_{\lambda}(A) d \lambda= \begin{cases}0 & \text { if } j<0 \\ I & \text { if } j=0 \\ A^{j} & \text { if } j>0\end{cases}
$$

where the integration is taken counter clockwise over a large circle enclosing $\sigma(A)$. From (10), (18), and (20) we find that

$$
Q_{0}=0, \quad Q_{1}=I,
$$

and

$$
Q_{j+1}=A^{j}-\sum_{p_{n} \leqq j}\left(\lambda_{n} E_{n}+F_{n}\right)^{j} \quad \text { if } j \geqq 1 .
$$

The series on the right in (22) is understood to mean

$$
\sum_{n=1}^{\infty} \varepsilon_{n, j}\left(\lambda_{n} E_{n}+F_{n}\right)^{j}
$$

where $\varepsilon_{n, j}=1$ if $p_{n} \leqq j$ and $\varepsilon_{n, j}=0$ if $j<p_{n}$.

It is part of the conclusion that the series in (23) is convergent in $[X]$. As a consequence, we have the following theorem:

THEOREm 2. If $A$ admits the uniform index $p$, the series

$$
\sum_{n=1}^{\infty}\left(\lambda_{n} E_{n}+F_{n}\right)^{j}
$$

converges when $p \leqq j$.

Concerning an absolute index sequence we have:

THeorem 3. If $\left\{p_{n}\right\}$ is an absolute index sequence for $A$, the series

$$
\sum_{n=1}^{\infty}\left\|\varepsilon_{n, j}\left(\lambda_{n} E_{n}+F_{n}\right)^{j}\right\|
$$

converges for each $j \geqq 1$.

Proof. We fix $j$ and suppose $\delta>0, \varepsilon>0$. Choose $N$ so large that $\left|\lambda_{k}\right|<\delta$ if $k \geqq N$ and also so large that 


$$
\sum_{n=r}^{s}\left\|S_{n}(\lambda)-P_{n}^{\left(p_{n}\right)}(\lambda)\right\|<\varepsilon \delta^{-(j+1)}
$$

if $N \leqq r<s$ and $|\lambda| \geqq \delta$. Since $\left|\lambda_{n}\right|<\delta, S_{n}(\lambda)-P_{n}^{\left(p_{n}\right)}(\lambda)$ can be calculated from (18) and (20):

$$
S_{n}(\lambda)-P_{n}^{\left(p_{n}\right)}(\lambda)=\sum_{k=p_{n}}^{\infty} \frac{\left(\lambda_{n} E_{n}+F_{n}\right)^{k}}{\lambda^{k+1}} .
$$

With integration in the counterclockwise sense over the circle on which $|\lambda|=\delta$ we have

$$
\varepsilon_{n, j}\left(\lambda_{n} E_{n}+F_{n}\right)^{j}=\frac{1}{2 \pi i} \oint \lambda^{j}\left[S_{n}(\lambda)-P_{n}^{\left(p_{n}\right)}(\lambda)\right] d \lambda .
$$

Putting $\lambda=\delta e^{i \theta}$ and using (26), we see that

$$
\sum_{n=r}^{s}\left\|\varepsilon_{n, j}\left(\lambda_{n} E_{n}+F_{n}\right)^{j}\right\| \leqq \frac{\delta^{j+1}}{2 \pi} \int_{0}^{2 \pi}\left\{\sum_{n=r}^{s}\left\|S_{n}(\lambda)-P_{n}^{\left(p_{n}\right)}(\lambda)\right\|\right\} d \theta<\varepsilon .
$$

This proves Theorem 3 .

Corollary. If $A$ admits the absolute index $p$, the series

$$
\sum_{n=1}^{\infty}\left\|\left(\lambda_{n} E_{n}+F_{n}\right)^{j}\right\|
$$

converges if $j \geqq p$.

3. Generalization of a theorem of Berkson. In this section we show how to construct an operator which has minimal uniform index 1 . The argument is modelled after the proof of a theorem due to E. R. Berkson (see $\S 1$ of Berkson [1]). Our theorem allows for multiple poles of the resolvent, whereas in Berkson's work there are simple poles only. We also discuss conditions for the operator to have minimal absolute index 1.

THEOREm 4. Let $\left\{\mu_{n}\right\}$ be a sequence of complex numbers with $\mu_{n} \neq 0$ and $\mu_{n} \rightarrow 0$. (We do not assume that $\mu_{m} \neq \mu_{n}$ if $m \neq n$.) Let $\left\{P_{n}\right\}$ and $\left\{R_{n}\right\}$ be sequences of elements in $[X]$ satisfying the conditions

$$
\begin{gathered}
P_{n} \neq 0, P_{n} P_{m}=0 \text { if } m \neq n, P_{n}^{2}=P_{n}, \\
P_{n} R_{n}=R_{n} P_{n}=R_{n}, \quad P_{n} R_{m}=R_{m} P_{n}=0 \text { if } m \neq n, \\
R_{m} R_{n}=0 \text { if } m \neq n .
\end{gathered}
$$

Finally, let $\left\{r_{n}\right\}$ be a sequence of positive integers, and suppose that

$$
R_{n}^{r_{n}}=0, \quad R_{n}^{r_{n}-1} \neq 0 .
$$


Now suppose that

$$
B=\sum_{n=1}^{\infty}\left(\mu_{n} P_{n}+R_{n}\right),
$$

it leing supposed that the series converges in $[X]$. Then $\sigma(B)$ consists of 0 and the distinct points among $\mu_{1}, \mu_{2}, \cdots$. The resolvent of $B$ is given by

$$
R_{\lambda}(B)=\frac{I}{\lambda}+\sum_{n=1}^{\infty}\left[T_{u}(\lambda)-\frac{P_{n}}{\lambda}\right]
$$

where

$$
T_{n}(\lambda)=\left\{\begin{array}{l}
\frac{P_{n}}{\lambda-\mu_{n}} \quad \text { if } r_{n}=1 \\
\frac{P_{n}}{\lambda-\mu_{n}}+\frac{R_{n}}{\left(\lambda-\mu_{n}\right)^{2}}+\cdots+\frac{R_{n}^{r_{n}-1}}{\left(\lambda-\mu_{n}\right)^{r_{n}}} \quad \text { if } r_{n}>1 .
\end{array}\right.
$$

If $\delta>0$ and if $m$ is such that $\left|\mu_{n}\right|<\delta$ when $n \geqq m$, the series

$$
\sum_{n=m}^{\infty}\left[T_{n}^{\prime}, \quad \frac{P_{n}}{\lambda}\right]
$$

converges uniformly when $|\lambda| \geqq \delta$. (This implies, in particular, that $R_{\lambda}(B)$ has a pole at $\mu_{n}$, so that $B$ is of meromorphic type.)

Proof. We write

$$
B_{n}=\sum_{k=1}^{n}\left(\mu_{k} P_{k}+R_{k}\right) .
$$

If $\lambda$ is fixed, different from $0, \mu_{1}, \cdots, \mu_{n}$, we define

$$
R_{n}(\lambda)=\frac{I}{\lambda}+\sum_{k=1}^{n}\left[T_{k}(\lambda)-\frac{P_{k}}{\lambda}\right] .
$$

The first step is to prove that $\sigma\left(B_{n}\right)$ is the set $\left\{0, \mu_{1}, \cdots, \mu_{n}\right\}$ and that $R_{n}(\lambda)$ is the resolvent of $B_{n}$. Direct calculations [which we omit-they are based on (28)-(30)] show that

$$
R_{n}(\lambda)\left(\lambda-B_{n}\right)=\left(\lambda-B_{n}\right) R_{n}(\lambda)=I
$$

if $\lambda$ is different from $0, \mu_{1}, \cdots, \mu_{n}$. Much as in the proof of Lemma 1 in $\S 2$ we prove that $\mu_{j}$ is an eigenvalue of $B_{n}$ if $1 \leqq j \leqq n$. To see that 0 is also an eigenvalue, let

$$
P=I-\left(P_{1}+\cdots+P_{n}\right),
$$

and observe that $P P_{n+1}=P_{n+1} \neq 0$, whence $P \neq 0$. Choose $y=P x$ so 
that $y \neq 0$, and observe that $B_{n} y=0$. This concludes the first step in the proof.

As the second step we apply a theorem of Newburgh [4]; since $B B_{n}=B_{n} B$ and $B_{n} \rightarrow B$, Newburgh's theorem enables us to conclude that $\sigma\left(B_{n}\right)$ converges to $\sigma(B)$ in the sense that the Hausdorff distance between the sets $\sigma(B)$ and $\sigma\left(B_{n}\right)$ approaches zero. In the present case this means that

$$
\sigma(B)=\bigcup_{n} \sigma\left(B_{n}\right)=\left\{0, \mu_{1}, \mu_{2}, \cdots\right\} .
$$

At the third step we obtain the formula (33) for the resolvent of $B$. When $\lambda \in \rho(B)$ we have

$$
\begin{aligned}
R_{n}(\lambda)(\lambda-B) & =R_{n}(\lambda)\left(\lambda-B_{n}\right)-R_{n}(\lambda)\left(B-B_{n}\right) \\
& =I-R_{n}(\lambda) \sum_{k=n+1}^{\infty}\left(\mu_{k} P_{k}+R_{k}\right),
\end{aligned}
$$

and a short calculation, using (28)-(30), yields

$$
R_{n}(\lambda)(\lambda-B)=I-\frac{I}{\lambda} \sum_{k=n+1}^{\infty}\left(\mu_{k} P_{k}+R_{k}\right) .
$$

If we multiply by $R_{\lambda}(B)$ and transpose, we obtain

$$
R_{\lambda}(B)-R_{n}(\lambda)=\frac{I}{\lambda} R_{\lambda}(B) \sum_{k=n+1}^{\infty}\left(\mu_{k} P_{k}+R_{k}\right) .
$$

We now pass to the limit as $n \rightarrow \infty$. Because of the assumed convergence of the series (32), we obtain the validity of (33) when $\lambda \in \rho(B)$.

The last step is the one concerning uniform convergence. Now, $\left\|\lambda^{-1} R_{\lambda}(B)\right\|$ is bounded on any subset of $\rho(B)$ in which the distance of $\lambda$ from $\sigma(B)$ has a positive lower bound. Consequently, we see from (36) that the convergence of $R_{n}(\lambda)$ to $R_{\lambda}(B)$ is uniform on any such subset of $\rho(B)$. This being established, suppose $\delta>0$, and let $m$ be such that $\left|\mu_{n}\right|<\delta$ if $n \geqq m$. Consider the operator

$$
\sum_{n=m}^{\infty}\left(\mu_{n} P_{n}+R_{n}\right) \text {. }
$$

We can apply the results proved thus far to it. Its spectrum is the set $\left\{0, \mu_{m}, \mu_{m+1}, \cdots\right\}$, and its resolvent is the series

$$
\frac{I}{\lambda}+\sum_{n=m}^{\infty}\left[T_{n}(\lambda)-\frac{P_{n}}{\lambda}\right]
$$

Hence, when $|\lambda| \geqq \delta$, the distance of $\lambda$ from the spectrum is positive, and so the series (37) converges uniformly when $|\lambda| \geqq \delta$. This completes the proof of Theorem 4. (To justify the parenthetical remark at the 
end of the statement of the theorem it suffices to look at formulas (34) and observe that, for fixed $n$, the number of indices $k$ such that $\mu_{k}=\mu_{n}$ is finite, because of the fact that $\mu_{k} \rightarrow 0$.)

If we add to the assumptions in Theorem 4 the assumption that $\mu_{n} \neq \mu_{m}$ if $n \neq m$, it is evident from the conclusions of the theorem that the residue of $R_{\lambda}(B)$ at $\mu_{n}$ is $P_{n}$. It then follows from the definition in $\S 1$ that $B$ is of minimal uniform index 1 relative to $\left\{\mu_{n}\right\}$.

At this point we can state a theorem giving an alternative criterion for an operator $A$ of meromorphic type to be of minimal uniform index 1 relative to a given enumeration of its poles.

THEOREM 5. Let $A$ be an operator of meromorphic type, and let the meanings of $\lambda_{n}, E_{n}, F_{n}$ be as in $\S 1$. Then a necessary and sufficient condition that $A$ have minimal uniform index 1 relative to $\left\{\lambda_{n}\right\}$ is that the series $\sum_{n=1}^{\infty}\left(\lambda_{n} E_{n}+F_{n}\right)$ converge in $[X]$.

Proof. The necessity of the condition is stated in Theorem 2. The sufficiency of the condition follows from Theorem 4, by taking $\mu_{n}=\lambda_{n}$, $P_{n}=E_{n}, R_{n}=F_{n}, r_{n}=q_{n}$ in Theorem 4 . Then, the assertion about the series (35) shows that the conditions for $A$ to admit the uniform index 1 are satisfied.

We now consider the effect of strengthening the hypotheses of Theorem 4.

THEOREM 6. To the assumptions of Theorem 4 we add the hypothesis that the series

$$
\sum_{n=1}^{\infty}\left\|\mu_{n} P_{n}+R_{n}\right\|
$$

is convergent. Then we can conclude that, if $\delta>0$ and if $m$ is chosen so that $\left|\mu_{k}\right|$ when $k \geqq m$, the series

$$
\sum_{n=m}^{\infty}|| T_{n}(\lambda)-\frac{P_{n}}{\lambda}||
$$

converges uniformly when $|\lambda| \geqq \delta$. Hence, in this case, if $\mu_{n} \neq \mu_{m}$ when $n \neq m$, the operator $B$ defined by (32) has the minimal absolute index 1.

Proof. From Lemma 1 we see that, when $\lambda$ is different from 0 and $\mu_{n}$,

$$
\left[\lambda-\left(\mu_{n} P_{n}+R_{n}\right)\right]^{-1}=\frac{I}{\lambda}+\left[T_{n}(\lambda)-\frac{P_{n}}{\lambda}\right] .
$$

This leads to the identity 


$$
T_{n}(\lambda)-\frac{P_{n}}{\lambda} \frac{1}{\lambda}\left\{\frac{I}{\lambda}+\left[T_{n}(\lambda)-\frac{P_{n}}{\lambda}\right]\right\}\left(\mu_{n} P_{n}+R_{n}\right) .
$$

Thus

$$
\left\|T_{n}(\lambda)-\frac{P_{n}}{\lambda}|| \leqq \frac{1}{|\lambda|}\left\{\frac{1}{|\lambda|}+\left\|T_{n}(\lambda)-\frac{P_{n}}{\lambda}\right\|\right\}\right\| \mu_{n} P_{n}+R_{n} \| .
$$

Now, as a consequence of the proven facts about the uniform convergence of the series (35), we can assert that the factor

$$
\frac{1}{|\lambda|}\left\{\frac{1}{|\lambda|}+\left\|T_{n}(\lambda)-\frac{P_{n}}{\lambda} \mid\right\|\right\}
$$

is bounded uniformly with respect to $n$ and $\lambda$ if $|\lambda| \geqq \delta$ and $n$ is sufficiently large. The asserted uniform convergence of (39) now follows from (40) and the assumed convergence of (38).

Just as we got Theorem 5 from Theorem 4 and Theorem 2, so we get the following theorem from Theorem 6 and the corollary of Theorem 3 .

Theorem 7. With $A, \lambda_{n}, E_{n}, F_{n}$ as in $\S 1, A$ has minimal absolute index 1 if and only if the series $\sum_{n=1}^{\infty}\left\|\lambda_{n} E_{n}+F_{n}\right\|$ converges.

With the assumptions of Theorem 6 it is not hard to see that $B$ has minimal absolute index 1 , even if the points $\mu_{1}, \mu_{2}, \cdots$ are not all distinct. Since $\mu_{n} \rightarrow 0$, there is only a finite set of $n$ 's for which $\mu_{n}=\mu_{1}$. We let $\lambda_{1}=\mu_{1}, E_{1}=$ the sum of the $P_{n}$ 's for which $\mu_{n}=\lambda_{1}, F_{1}=$ the sum of the corresponding $R_{n}$ 's. Then let $\lambda_{2}$ be the first $\mu_{n}$ different from $\lambda_{1}$, and continue in the obvious way. It turns out that the pole at $\lambda_{1}$ is of order $q_{1}$, where $q_{1}$ is the largest of the $r_{n}$ 's corresponding to $\mu_{n}$ 's with $\mu_{n}=\lambda_{1}$. Likewise for the pole at $\lambda_{2}$, and so on. Because of the convergence of (38) we see that

$$
B=\sum_{n=1}^{\infty}\left(\lambda_{n} E_{n}+F_{n}\right)
$$

with

$$
\sum_{n=1}^{\infty}\left\|\lambda_{n} E_{n}+F_{n}\right\|<\infty \text {. }
$$

Theorem 6 now applies, with $\lambda_{n}, E_{n}, F_{n}, q_{n}$ in place of $\mu_{n}, P_{n}, R_{n}, r_{n}$. (We leave to the reader the verification that the required conditions on $E_{n}$ and $F_{n}$, corresponding to $(28)-(31)$, are satisfied.)

4. Operators of minimal uniform index 1. We return now to the notations of $\S \S 1$ and 2 . Suppose that $A$ is of meromorphic type, with minimal uniform index 1 relative to $\left\{\lambda_{n}\right\}$. By (10), (19), (21) and (22) we 
see that

$$
\begin{aligned}
R_{\lambda}(A)=\frac{I}{\lambda} & +\sum_{n=1}^{\infty}\left[S_{n}(\lambda)-\frac{E_{n}}{\lambda}\right] \\
& +\sum_{j=1}^{\infty} \frac{1}{\lambda^{j+1}}\left[A^{\jmath}-\sum_{n=1}^{\infty}\left(\lambda_{n} E_{n}+F_{n}\right)^{\jmath}\right] .
\end{aligned}
$$

Let us define an operator $B$ by the formula

$$
B=\sum_{n=1}^{\infty}\left(\lambda_{n} E_{n}+F_{n}\right) .
$$

We know from Theorem 4 that $\sigma(B)=\sigma(A)$, that

$$
R_{\lambda}(B)=\frac{I}{\lambda}+\sum_{n=1}^{\infty}\left[S_{n}(\lambda)-\frac{E_{n}}{\lambda}\right] \text {, }
$$

and that $B$ is also an operator of meromorphic type with minimal uniform index 1 relative $\left\{\lambda_{n}\right\}$. We also see, from Theorem 7 , that if $A$ has minimal absolute index 1 , the same is true of $B$.

When $B$ is related to $A$ by (42) under the circumstances here described, and when it turns out that $B=A$, we shall say that $A$ is canonical relative to $\left\{\lambda_{n}\right\}$. The reader is reminded that $E_{n}$ is the residue of $R_{\lambda}(A)$ at $\lambda_{n}$ and that $F_{n}=\left(A-\lambda_{n}\right) E_{n}$.

Since $F_{n} E_{n}=F_{n}$, it is clear from (42) that $\left(B-\lambda_{n}\right) E_{n}=F_{n}$. From (43) we see that the residue of $R_{\lambda}(B)$ at $\lambda_{n}$ is $E_{n}$. Hence the $B$ given by (42) is canonical relative to $\left\{\lambda_{n}\right\}$, even if $A$ is not. We may refer to $B$ as the canonical part of $A$ relative to $\left\{\lambda_{n}\right\}$. This naturally prompts us to investigate the nature of the operator $A-B$, which we denote by $C$.

Teeorem 8. If $A$ has minimal uniform index 1 relative to $\left\{\lambda_{n}\right\}$, and if $B$ is the canonical xart of $A$ relative to $\left\{\lambda_{n}\right\}$, the operator $C=A-B$ is quasinilpotent $[\sigma(C)=(0)]$, and $B C=C B=0$.

Proof. From $\lambda_{n} E_{n}+F_{n}=A E_{n}$ we see that

$$
B=\sum_{n=1}^{\infty} A E_{n} \text { and } B E_{m}=A E_{m} .
$$

Then (because $A B=B A$ )

$$
A B=\sum_{n=1}^{\infty} A^{2} E_{n}, \quad B^{2}=\sum_{n=1}^{\infty} A B E_{n}=A B,
$$

whence $B C=C B=A B-B^{2}=0$. It now follows easily by induction that $A^{n}=B^{n}+C^{n}$. It also follows from (42) by induction that

$$
B^{j}=\sum_{n=1}^{\infty}\left(\lambda_{n} E_{n}+F_{n}\right)^{j}, \quad j=1,2, \cdots .
$$


On referring back to (41) and (43) we now see that

$$
R_{\lambda}(A)=R_{\lambda}(B)+\sum_{j=1}^{\infty} \frac{C^{j}}{\lambda^{j+1}} .
$$

The series here is convergent if $\lambda \neq 0$. This implies that $C$ is quasinilpotent, i.e. that $\sigma(C)$ consists of 0 alone, or that $\left\|C^{n}\right\|^{1 / n} \rightarrow 0$. This completes the proof.

The foregoing considerations raise the question as to whether the decompsition $A=B+C$ is unique, in the following sense. Is it conceivable that we could write $A=B_{1}+C_{1}$, where $C_{1}$ is quasinilpotent, $B_{1} C_{1}=$ $C_{1} B_{1}=0$, and $B_{1}$ is an operator of meromorphic type which is of minimal uniform index 1 and canonical, relative to some enumeration of the poles of $R_{\lambda}\left(B_{1}\right)$, but such that $B_{1}$ is not the canonical part of $A$ relative to an enumeration of the poles of $R_{\lambda}(A)$ ? The answer to this question is negative, as we now show.

THEOREM 9. Let $B_{1}$ be of meromorphic type, of minimal uniform index 1 and canonical relative to an enumeration $\left\{\mu_{n}\right\}$ of the distinct poles of $R_{\lambda}\left(B_{1}\right)$. Let $P_{n}$ be the residue of $R_{\lambda}\left(B_{1}\right)$ at $\mu_{n}$, and let $R_{n}=$ $\left(B_{1}-\mu_{n}\right) P_{n}$. Let $C_{1}$ be a quasinilpotent operator such that $B_{1} C_{1}=$ $C_{1} B_{1}=0$. Let $A_{1}=B_{1}+C_{1}$. Then $A_{1}$ is of meromorphic type with $\sigma\left(A_{1}\right)=\sigma\left(B_{1}\right) ; A_{1}$ has minimal uniform index 1 relative to $\left\{\mu_{n}\right\}$, and $B_{1}$ is the canonical part of $A_{1}$ relative to $\left\{\mu_{n}\right\}$.

Proof. From $B_{1} C_{1}=C_{1} B_{1}=0$ we deduce that $A_{1} B_{1}=B_{1}^{2}=B_{1} A_{1}$. From general spectral theory we know that $\mu_{n}$ is an eigenvalue of $B_{1}$, so there exists $x_{n}$ for which $B_{1} x_{n}=\mu_{n} x_{n} \neq 0$. Then $A_{1} B_{1} x_{n}=B_{1}^{2} x_{n}=$ $B_{1}\left(\mu_{n} x_{n}\right)=\mu_{n} B_{1} x_{n}$, so that $\mu_{n}$ is also an eigenvalue of $A_{1}$. Since the spectrum is closed, it follows that $\sigma\left(B_{1}\right) \subset \sigma\left(A_{1}\right)$. Next, we show that $\rho\left(B_{1}\right) \subset \rho\left(A_{1}\right)$ and that

$$
R_{\lambda}\left(A_{1}\right)=R_{\lambda}\left(B_{1}\right)+\sum_{n=1}^{\infty} \frac{C_{1}^{n}}{\lambda^{n+1}}
$$

if $\lambda \in \rho\left(B_{1}\right)$. It will then follow that $\sigma\left(A_{1}\right)=\sigma\left(B_{1}\right)$.

To begin with, if $|\lambda|>\left\|B_{1}\right\|$ we know that

$$
R_{\lambda}\left(B_{1}\right)=\sum_{n=0}^{\infty} \frac{B_{1}^{n}}{\lambda^{n+1}} .
$$

It is then an easy calculation to show that

$$
\left(\lambda-A_{1}\right)\left[R_{\lambda}\left(B_{1}\right)+\sum_{n=1}^{\infty} \frac{C_{1}^{n}}{\lambda^{n+1}}\right]=I
$$


if $|\lambda|>\left\|B_{1}\right\|$. Because $\rho\left(B_{1}\right)$ is a connected set, and the left member of (46) is an analytic function on $\rho\left(B_{1}\right)$, it follows that (46) is valid for each $\lambda$ in $\rho\left(B_{1}\right)$. The same argument applies with the order of the factors reversed. Hence we conclude that $\rho\left(B_{1}\right) \subset \rho\left(A_{1}\right)$ and that (45) holds.

From the form of (45) and the assumption on $B_{1}$ it follows that $\mu_{n}$ is a pole of $R_{\lambda}\left(A_{1}\right)$, the residue there being the same as the residue of $R_{\lambda}\left(B_{1}\right)$, namely $P_{n}$. In fact, we can also see that when we calculate the Laurent series for each of $R_{\lambda}\left(A_{1}\right)$ and $R_{\lambda}\left(B_{1}\right)$ in powers of $\lambda-\mu_{n}$, we get the same terms for both in the case of negative powers of $\lambda-\mu_{n}$. In particular, the coefficient of $\left(\lambda-\mu_{n}\right)^{-2}$ in one case is $\left(A_{1}-\mu_{n}\right) P_{n}$, and in the other case it is $\left(B_{1}-\mu_{n}\right) P_{n}=R_{n}$. Therefore $R_{n}=\left(A_{1}-\mu_{n}\right) P_{n}$. It is now clear that $A_{1}$ is of minimal uniform index 1 relative to $\left\{\mu_{n}\right\}$ and that $B_{1}$ is the canonical part of $A_{1}$.

5. Operators of minimal uniform index $p$. Returning once more to the notation of $\S 1$, let us assume that $A$ is of meromorphic type and that it has minimal uniform index $p$ relative to $\left\{\lambda_{n}\right\}$. We are now interested in the case when $p>1$. By (10), (21) and (22) we see that

$$
\begin{aligned}
R_{\lambda}(A)=\frac{I}{\lambda}+\frac{A}{\lambda^{2}}+\cdots & +\frac{A^{p-1}}{\lambda^{p}}+\sum_{n=1}^{\infty}\left[S_{n}(\lambda)-P_{n}^{(p)}(\lambda)\right] \\
& +\sum_{j=p}^{\infty} \frac{1}{\lambda^{j+1}}\left[A^{j}-\sum_{n=1}^{\infty}\left(\lambda_{n} E_{n}+F_{n}\right)^{j}\right] .
\end{aligned}
$$

This formula takes an especially simple form if

$$
A^{p}=\sum_{n=1}^{\infty}\left(\lambda_{n} E_{n}+F_{n}\right)^{p}
$$

because

$$
\lambda_{n} E_{n}+F_{n}=A E_{n},\left(\lambda_{n} E_{n}+F_{n}\right)^{j}=A^{\prime} E_{n},
$$

so that

$$
A^{p}=\sum_{n=1}^{\infty} A^{p} E_{n}
$$

whence it follows that

$$
A^{j}=\sum_{n=1}^{\infty} A^{\jmath} E_{n} \quad \text { if } j \geqq p,
$$

and so (47) becomes, in this special case,

$$
R_{\lambda}(A)=\frac{I}{\lambda}+\frac{A}{\lambda^{2}}+\cdots+\frac{A^{p-1}}{\lambda^{p}}+\sum_{n=1}^{\infty}\left[S_{n}(\lambda)-P_{n}^{(x)}(\lambda)\right] .
$$

When $A$ is of minimal uniform index $p$ relative to $\left\{\lambda_{n}\right\}$, and when 
(48) holds, we say that $A$ is canonical of order $p$ relative to $\left\{\lambda_{n}\right\}$. In $\S 7$ we discuss an unsolved problem involving the concept of a canonical operator of order $p$, for the case in which $A$ itself is not canonical.

We now present a generalization of Theorem 5 .

THEOREM 10. Let $A$ be of meromorphic type, and let $\lambda_{n}, E_{n}, F_{n}$ be as in $\S 1$. Suppose that $p$ is a positive integer, and suppose that the series $\sum_{n=1}^{\infty}\left(\lambda_{n} E_{n}+F_{n}\right)^{p}$ is convergent in $[X]$. Then $A$ admits the uniform index p relative to $\left\{\lambda_{n}\right\}$. Hence (see Theorem 2), in order that $A$ have minimal uniform index $p$-relative to $\left\{\lambda_{n}\right\}$, it is neceseary and sufficient that $\sum_{n=1}^{\infty}\left(\lambda_{n} E_{n}+F_{n}\right)^{p}$ converge in $[X]$ and that $\sum_{n=1}^{\infty}\left(\lambda_{n} E_{n}+F_{n}\right)^{p-1}$ not converge in $[X]$.

Proof. Our argument is valid if $p \geqq 1$. Hence this theorem includes Theorem 5; the proof is different from that of Theorem 5, however, even when $p=1$. The argument hinges on two identities. Let $H_{n}=\lambda_{n} E_{n}+F_{n}$. Then

$$
S_{n}(\lambda)-P_{n}^{(p)}(\lambda)=\lambda^{-p} R_{\lambda}\left(H_{n}\right) H_{n}^{p}
$$

if $\lambda \neq 0$ and $\lambda \neq \lambda_{n}$. The other identity is

$$
R_{\lambda}\left(H_{n}\right) H_{n}^{p}=R_{\lambda}(A) H_{n}^{p} ;
$$

it is valid if $\lambda \in \rho(A)$. To prove (50) we may assume $|\lambda|>\left|\lambda_{n}\right|$. When (50) has been proved under this condition the general assertion about the validity of (50) follows by the principle of analytic continuation, because the expressions on each side of the equality in (50) are analytic in $\lambda$ except at 0 and $\lambda_{n}$. (We see by Lemma 1 in $\S 2$ that $\sigma\left(H_{n}\right)=\left\{0, \lambda_{n}\right\}$.) Now, from (18) and (20) we see that

$$
S_{n}(\lambda)-P_{n}^{(p)}(\lambda)=\sum_{k=p}^{\infty} \frac{H_{n}^{k}}{\lambda^{k+1}} \quad \text { if }|\lambda|>\left|\lambda_{n}\right| .
$$

From the Neumann series for $R_{\lambda}\left(H_{n}\right)$ we see that

$$
\lambda^{-p} R_{\lambda}\left(H_{n}\right) H_{p}^{n}=\frac{H_{n}^{p}}{\lambda^{p}} \sum_{\jmath=0}^{\infty} \frac{H_{n}^{j}}{\lambda^{j+1}}=\sum_{k=p}^{\infty} \frac{H_{n}^{k}}{\lambda^{k+1}} .
$$

Thus (50) is proved.

In proving (51), because of the principle of analytic continuation, we may restrict ourselves to values of $\lambda$ so large that $\left\|A-\lambda_{n}\right\|<\left|\lambda-\lambda_{n}\right|$. With this restriction, noting that $\lambda-A=\left(\lambda-\lambda_{n}\right)-\left(A-\lambda_{n}\right)$, we can write

$$
R_{\lambda}(A)=\sum_{k=0}^{\infty} \frac{\left(A-\lambda_{n}\right)^{k}}{\left(\lambda-\lambda_{n}\right)^{k+1}} .
$$


Now from $F_{n}=\left(A-\lambda_{n}\right) E_{n}, H_{n}=\lambda_{n} E_{n}+F_{n}$, and $E_{n}^{2}=E_{n}$, we deduce that $H_{n} F_{n}=\left(A-\lambda_{n}\right) H_{n}$. It then follows readily that $H_{n}^{p} F_{n}^{k}=\left(A-\lambda_{n}\right)^{k} H_{n}^{p}$ for all positive integers $p$ and $k$. Since $F_{n}^{k}=0$ if $k \geqq q_{n}$, it follows from (52) that

$$
R_{\lambda}(A) H_{n}^{p}=\sum_{k=0}^{q_{n}-1} \frac{H_{n}^{p} F_{n}^{k}}{\left(\lambda-\lambda_{n}\right)^{k+1}} .
$$

On the other hand, we know from (15) that

$$
R_{\lambda}\left(H_{n}\right)=\frac{I-E_{n}}{\lambda}+\frac{E_{n}}{\lambda-\lambda_{n}}+\frac{F_{n}}{\left(\lambda-\lambda_{n}\right)^{2}}+\cdots+\frac{F_{n}^{q_{n}-1}}{\left(\lambda-\lambda_{n}\right)^{q_{n}}} ;
$$

because $H_{n}^{p} E_{n}=H_{n}^{p}$, we see from this and (53) that (51) holds.

We now turn to the proof of Theorem 10. We have to show that the convergence of the series $\sum_{n=1}^{\infty} H_{n}^{p}$ implies that the series

$$
\sum_{n=m}^{\infty}\left[S_{n}(\lambda)-P_{n}^{(p)}(\lambda)\right]
$$

converges uniformly when $|\lambda| \geqq \delta$, provided merely that $\delta>0$ and that $m$ is chosen in such a way that $\left|\lambda_{k}\right|<\delta$ when $k \geqq m$. When $\delta$ and $m$ are specified, there may perhaps be a finite set of $\lambda_{i}$ 's for which $\left|\lambda_{i}\right| \geqq \delta$. We can diminish $\delta$ slightly, if necessary, so as to have $\left|\lambda_{i}\right|>\delta$ for all these $\lambda_{\imath}^{\prime}$ s and still have $\left|\lambda_{k}\right|<\delta$ if $k \geqq m$. If we give the proof with this modified $\delta$, that is certainly sufficient. Choose a positive number $\alpha$ so small that, if $\left|\lambda_{i}\right|>\delta$, the closed circular disk of radius $\alpha$ and center $\lambda_{i}$ lies entirely outside the circle on which $|\lambda|=\delta$, and so that any two such disks do not touch or overlap. Let $E(\delta, \alpha)$ be the set of $\lambda$ 's such that $|\lambda| \geqq \delta$ and $\left|\lambda-\lambda_{i}\right|=\alpha$ if $\left|\lambda_{i}\right|>\delta$. There is some constant $M$ such that $\left\|\lambda^{-p} R_{\lambda}(A)\right\| \leqq M$ if $\lambda \in E(\delta, \alpha)$. Now, suppose that $\varepsilon>0$. By hypothesis we can choose an integer $N$ so large that

$$
M|| \sum_{n=j}^{k} H_{n}^{p}||<\varepsilon
$$

if $N \leqq j \leqq k . \quad B y(50)$ and (51) we can write

$$
\sum_{n=j}^{k}\left[S_{n}(\lambda)-P_{n}^{(p)}(\lambda)\right]=\lambda^{-p} R_{\lambda}(A) \sum_{n=j}^{k} H_{n}^{p},
$$

provided that $\lambda \in \rho(A)$. Therefore certainly

$$
\|\left|\sum_{n=j}^{k}\left[S_{n}(\lambda)-P_{n}^{(p)}(\lambda)\right]\right| \mid<\varepsilon
$$

if $N \leqq j \leqq k$, provided that $\lambda \in E(\delta, \alpha)$. In fact, however, (54) holds whenever $|\lambda| \geqq \delta$; that is, it holds even if $|\lambda| \geqq \delta,\left|\lambda_{i}\right|>\delta$, and $\left|\lambda-\lambda_{i}\right|<\alpha$. For, the function

$$
\sum_{n=j}^{k}\left[S_{n}(\lambda)-P_{n}^{(p)}(\lambda)\right]
$$


is holomorphic in $\lambda$ when $\left|\lambda-\lambda_{i}\right| \leqq \alpha$, and its norm is less than $\varepsilon$ when $\left|\lambda-\lambda_{i}\right|=\alpha$; therefore the norm is also less than $\varepsilon$ when $\left|\lambda-\lambda_{i}\right|<\alpha$, by the maximum modulus theorem, which is valid for analytic functions with values in a complex Banach space (see Taylor [5], Theorem 4. 42-A, or Hille-Phillips [3], p. 100). This completes the proof of Theorem 10.

In the next theorem we present information about the operator $A^{p}$ under the assumption that $A$ has minimal uniform index $p$.

THEOREM 11. Suppose that $A$ is of meromorphic type and of minimal uniform index $p$ relative to $\left\{\lambda_{n}\right\}$, with $p>1$. (We adopt the notation of $\S 1$ as regards $\lambda_{n}, E_{n}, F_{n}$.) Define operators $L, M$ by the formulas

$$
L=\sum_{n=1}^{\infty}\left(\lambda_{n} E_{n}+F_{n}\right)^{p}, \quad M=A^{p}-L
$$

Let

$$
\begin{gathered}
G_{n}=\left(\lambda_{n} E_{n}+F_{n}\right)^{p}-\lambda_{n}^{p} E_{n}, \\
V_{n}(\lambda)=\left\{\begin{array}{l}
\frac{E_{n}}{\lambda-\lambda_{n}^{p}} \text { if } q_{n}=1 \\
\frac{E_{n}}{\lambda-\lambda_{n}^{p}}+\frac{G_{n}}{\left(\lambda-\lambda_{n}^{\prime}\right)^{2}}+\cdots+\frac{G^{q_{n}-1}}{\left(\lambda-\lambda_{n}^{\gamma}\right)^{q_{n}}} .
\end{array}\right.
\end{gathered}
$$

Then $L$ is an operator of meromorphic type with $\sigma(L)$ consisting of $\lambda_{1}^{p}, \lambda_{2}^{p}, \cdots$ aud 0 . The resolvent of $L$ is

$$
R_{\lambda}(L)=\frac{I}{\lambda}+\sum_{n=1}^{\infty}\left[V_{n}(\lambda)-\frac{E_{n}}{\lambda}\right] \text {. }
$$

If $\delta>0$ and if $m$ is such that $\left|\lambda_{n}^{p}\right|<\delta$ when $n \geqq m$, then the series

$$
\sum_{n=m}^{\infty}\left[V_{n}(\lambda)-\frac{E_{n}}{\lambda}\right]
$$

converges uniformly when $|\lambda| \geqq \delta$. The operator $M$ is quasinilpotent, and $L M=M L=0$. Finally, if all the points $\lambda_{1}^{p}, \lambda_{2}^{p}, \cdots$ are distinct, $A^{k}$ has minimal uniform index 1 relative to $\left\{\lambda_{n}^{p}\right\}$, and $L$ is the canonical part of $A^{p}$ relative to $\left\{\lambda_{n}^{p}\right\}$.

Proof. By expanding $\left(\lambda_{n} E_{n}+F_{n}\right)^{p}$, we see from (56) that $G_{n}$ can be expressed in the form $G_{n}=F_{n} J_{n}=J_{n} F_{n}$, where $J_{n} \in[X]$. It is then easy to see that $E_{n} G_{n}=G_{n} E_{n}=G_{n}$ and $G_{n}^{q_{n}}=0$. We shall show that $G_{n}^{q_{n}-1} \neq 0$ if $q_{n}>$ I. In fact, from (56) we can write $G_{n}=p \lambda_{n}^{p-1} F_{n}+F_{n}^{2} K_{n}$, where $K_{n} \in[X]$ and $F_{n} K_{n}=K_{n} F_{n}$. Thus $G_{n}^{q_{n}-1}=0$ would imply

$$
F_{n}^{q_{n}-1}\left[p \lambda_{n}^{p-1} I+F_{n} K_{n}\right]^{q_{n}-1}=0 .
$$


Since $F_{n}^{q_{n}}=0$ and $p \lambda_{n}^{p-1} \neq 0$, this would imply $F_{n}^{q_{n}-1}=0$, contrary to fact.

We can now apply Theorem 4 , taking $\mu_{n}=\lambda_{n}^{p}, P_{n}=E_{n}, R_{n}=G_{n}, r_{n}=q_{n}$. From (55) and (56) we see that

$$
L=\sum_{n=1}^{\infty}\left(\lambda_{n}^{p} E_{n}+G_{n}\right) ;
$$

therefore we obtain at once our assertions about $\sigma(L)$ and $R_{\lambda}(L)$. The convergence behavior of the series (58) insures that $R_{\lambda}(L)$ has a pole at $\lambda_{n}^{p}$.

Now consider the operator $M$ defined in (55). From (2) we see that $\dot{A} E_{n}=\lambda_{n} E_{n}+F_{n}$, so we can write

$$
L=\sum_{n=1}^{\infty} A^{p} E_{n},
$$

from which it follows that $L A^{p}=A^{p} L=L^{2}$. Then $L M=L\left(A^{p}-L\right)=0$, and likewise $M L=0$. It follows that $A^{n p}=L^{n}+M^{n}, n=1,2, \cdots$. To prove that $M$ is quasinilpotent we shall show that the series

$$
\sum_{n=1}^{\infty} \frac{M^{n}}{\lambda^{n+1}}
$$

converges if $\lambda \neq 0$. Now, from (55) it follows readily that

$$
L^{j}=\sum_{n=1}^{\infty}\left(\lambda_{n} E_{n}+F_{n}\right)^{i n} \quad \text { if } j \geqq 1 .
$$

Hence, what we wish to prove is that the series

$$
\sum_{j=1}^{\infty} \frac{A^{j p}-\sum_{n=1}^{\infty}\left(\lambda_{n} E_{n}+F_{n}\right)^{j p}}{\lambda^{j+1}}
$$

is convergent if $\lambda \neq 0$. We know from (47) that the series

$$
\sum_{k=p}^{\infty} \frac{A^{k}-\sum_{n=1}^{\infty}\left(\lambda_{n} E_{n}+F_{n}\right)^{k}}{\lambda^{j+1}}
$$

converges, and from this we can deduce the convergence of the series in (59). If $\lambda \neq 0$, choose a circle of radius $\varepsilon$ centered at 0 , where $0<\varepsilon, \varepsilon^{p}<|\lambda|$. Then, integration around this circle in the counterclokwise sense yields

$$
\frac{1}{2 \pi i} \oint \frac{d t}{\left(\lambda-t^{p}\right) t^{k+1}}=\left\{\begin{array}{cl}
\frac{1}{\lambda^{k+1}} & \text { if } k=j p, j=1,2, \cdots \\
0 & \text { if } k \text { is not a multiple of } p .
\end{array}\right.
$$

Therefore, if

$$
D_{k}=A^{k}-\sum_{n=1}^{\infty}\left(\lambda_{n} E_{n_{r}}+F_{n}\right)^{h}
$$


we have

$$
\frac{1}{2 \pi i} \oint \frac{1}{\lambda-t^{p}}\left(\sum_{k=p}^{\infty} \frac{D_{k}}{t^{k+1}}\right) d t=\sum_{j=1}^{\infty} \frac{D_{j p}}{\lambda^{j+1}},
$$

the last series on the right being convergent. By comparison with (59), we see that we have finished the proof that $M$ is quasinilpotent.

It is clear from the situation that $L$ is of minimal uniform index 1 and that $L$ is canonical relative to $\left\{\lambda_{n}^{p}\right\}$ when the points $\lambda_{1}^{p}, \lambda_{2}^{p}, \cdots$ are all distinct. The final assertion of Theorem 11 is then a consequence of Theorem 9 .

6. Operators of minimal absolute index $p$. Suppose that $A$ is of meromorphic type and of minimal absolute index $p$. We follow the notation of $\S 1$. It is then certainly true that $A$ admits the uniform index $p$ relative to $\left\{\lambda_{n}\right\}$, although $p$ is not necessarily the minimal uniform index. Let $L, M$, and $G_{n}$ be defined by (55) and (56). By the corollary of Theorem 3 we know that the series

$$
\sum_{n=1}^{\infty}\left\|\lambda_{n}^{p} E_{n}+G_{n}\right\|
$$

is convergent. From the remarks at the end of $\S 3$ we see that $L$ has minimal absolute index 1 , regardless of whether or not the point $\lambda_{1}^{p}, \lambda_{2}^{p}, \cdots$ are all distinct. Under our present assumptions the operator $M$ defined in (55) is quasinilpotent, for the reasoning about this in the proof of Theorem 11 is all applicable. It is obvious that $L$ is of minimal uniform index 1 and canonical relative to any enumeration of the distinct poles of $R_{\lambda}(L)$. It then follows as in Theorem 9 and its proof that $\sigma\left(A^{p}\right)=\sigma(L)$ and that

$$
R_{\lambda}\left(A^{p}\right)=R_{\lambda}(L)+\sum_{n=1}^{\infty} \frac{M_{n}}{\lambda^{n+1}} \quad \text { if } \lambda \in \sigma\left(A^{p}\right)
$$

From (60) and the definitions in $\S 1$ we can then see that $A^{p}$ is of meromorphic type and minimal absolute index 1 . We state all this formally:

THEOREM 12. If $A$ is of meromorphic type and minimal absolute index $p, A^{p}$ is of meromorphic type and minimal absolute index 1.

The following theorem is analogous to Theorem 10 .

THEOREM 13. Let $A$ be of meromorphic type, and let the notation of $\S 1$ be adopted. Then $A$ admits the absolute index $p$ if and only if the series 


$$
\sum_{n=1}^{\infty}\left\|\left(\lambda_{n} E_{n}+F_{n}\right)^{p}\right\|
$$

is convergent. Hence, $A$ has minimal absolute index $p$ (a positive integer) if and only if

$$
\sum_{n=1}^{\infty}\left\|\left(\lambda_{n} E_{n}+F_{n}\right)^{p}\right\|<\infty \text { and } \sum_{n=1}^{\infty}\left\|\left(\lambda_{n} E_{n}+F_{n}\right)^{p-1}\right\|=\infty .
$$

Proof. In view of the corollary of Theorem 3, we have only to prove that $A$ admits the absolute index $p$ when the series (61) is convergent.

We begin by calling attention once more to (50) and (51), and to the notation $H_{n}=\lambda_{n} E_{n}+F_{n}$. We are supposing that

$$
\sum_{n=1}^{\infty}\left\|H_{n}^{p}\right\|<\infty \text {. }
$$

Suppose $\delta>0$, and let $m$ be such that $\left|\lambda_{k}\right|<\delta$ if $k \geqq m$. We assert the existence of a positive number $C$ that

$$
\left\|R_{\lambda}\left(H_{n}\right) H_{n}^{p}\right\| \leqq C\left\|H_{n}^{p}\right\|
$$

if $|\lambda| \geqq \delta$ and $n \geqq m$. Once this is proved, the fact that

$$
\sum_{n=m}^{\infty}\left\|S_{n}(\lambda)-P_{n}^{(p)}(\lambda)\right\|
$$

converges uniformly when $|\lambda| \geqq \delta$ will follow from (50) and (62), and our proof will be accomplished. There is only a finite set of $k$ 's for which $\left|\lambda_{k}\right| \geqq \delta$. Choose $\varepsilon>0$ so small that $\lambda \in \rho(A)$ if $0<\left|\lambda-\lambda_{k}\right| \leqq \varepsilon$ for these $k$ 's. We know that $\left\|R_{\lambda}(A)\right\|$ is bounded by some positive constant $C_{0}$ when $\lambda$ is at a distance greater than or equal to $\varepsilon$ from $\sigma(A)$. Consider the situation if $0<\left|\lambda-\lambda_{k}\right| \leqq \varepsilon$ and $\left|\lambda_{k}\right| \geqq \delta$. We know there is a function of $\lambda$ with values in $[X]$, call it $f_{k}$, such that $f_{k}$ is analytic when $\left|\lambda-\lambda_{k}\right| \leqq \varepsilon$ and

$$
R_{\lambda}(A)=f_{k}(\lambda)+S_{k}(\lambda)
$$

if $0<\left|\lambda-\lambda_{k}\right| \leqq \varepsilon$. Now $H_{n}^{p} E_{k}=H_{n}^{p} F_{k}=0$ if $n \neq k$. Therefore, in view of the definition of $S_{k}(\lambda)$ in section $1, R_{\lambda}(A) H_{n}^{p}=f_{k}(\lambda) H_{n}^{p}$, and we see from (51) that $\left\|R_{\lambda}\left(H_{n}\right) H_{n}^{p}\right\|=\left\|f_{k}(\lambda) H_{n}^{p}\right\|$ will have a bound $C_{k}\left\|H_{n}^{p}\right\|$ if $\left|\lambda-\lambda_{k}\right| \leqq \varepsilon$. If we choose $C$ at least as large as $C_{0}$ and the largest of the finite set of $C_{k}^{\prime} s$, (62) will hold. This ends the proof.

7. An open problem. Suppose that $A$ is of meromorphic type and of minimal uniform index $p$ relative to $\left\{\lambda_{n}\right\}$, where $p>1$. Suppose further that $A$ is not canonical of order $p$ relative to $\left\{\lambda_{n}\right\}$ (see $\S 5$ ). Is there in this case some uniquely determined operator $B$ which deserves 
to be called the canonical part of $A$ relative to $\left\{\lambda_{n}\right\}$ ? For the case $p=1$ we know that this question has an affirmative answer. The details are given in $\S 4$; the required $B$ is given by (42). For the case $p>1$, however, we have been unable to answer the question. We are even unable to say with certainty what properties should characterize the required $B$. We would certainly want $B$ to have the same spectrum as $A$, and for each $\lambda_{n}$ we would want the Laurent expansion of $R_{\lambda}(B)$ in powers of $\lambda-\lambda_{n}$ to consist of $S_{n}(\lambda)$ plus a series of nonnegative powers of $\lambda-\lambda_{n}$. We would also require that

$$
B^{p}=\sum_{n=1}^{\infty}\left(\lambda_{n} E_{n}+F_{n}\right)^{p}
$$

The requirements thus far stated would be sufficient to imply that $B$ is of minimal uniform index $p$ and canonical of order $p$ relative to $\left\{\lambda_{n}\right\}$, with

$$
R_{\lambda}(B)=\frac{I}{\lambda}+\frac{B}{\lambda^{2}}+\cdots+\frac{B^{p-1}}{\lambda^{p}}+\sum_{n=1}^{\infty}\left[S_{n}(\lambda)-P_{n}^{(p)}(\lambda)\right]
$$

when $\lambda \in \rho(B)$. It is not clear, however that there is at most one $B$ fulfilling all these conditions. Nor is there, so far as we can see, any method of proving the existence of a $B$ with these properties.

One possible starting point might be to define

$$
B_{n}=\sum_{k=1}^{n}\left(\chi_{k} E_{k}+F_{k}\right) \text {. }
$$

It may be proved that $\sigma\left(B_{n}\right)=\left\{0, \lambda_{1}, \cdots, \lambda_{n}\right\}$ and that

$$
R_{\lambda}\left(B_{n}\right)=\frac{I}{\lambda}+\frac{B_{n}}{\lambda^{2}}+\cdots+\frac{B_{n}^{p-1}}{\lambda^{p}}+\sum_{k=1}^{n}\left[S_{k}(\lambda)-P_{n}^{(p)}(\lambda)\right] .
$$

But, if $p>1, B_{n}$ has no limit in the sense of convergence in $[X]$. It is true that

$$
B_{n}^{p} \rightarrow \sum_{k=1}^{\infty}\left(\lambda_{k} E_{k}+F_{k}\right)^{p} .
$$

What appears to be needed is some way of assigning a limit $B$ to the sequence $\left\{B_{n}\right\}$ is some generalized sense, and then proving that this limit $B$ is the unique operator with the required properties. In particular cases it may (and does) happen that we can define $B$ by $B x=\lim _{n \rightarrow \infty} B_{n} x$ for each $x$. But we have no general theory.

8. Examples. In this concluding section we show how to construct an operator $A$ with minimal uniform index 1 or 2 such that $A$ either admits no absolute index at all, or admits an arbitrarily prescribed minimal absolute index (greater than or equal to the minimal uniform 
index, of course).

We also show how to construct an operator $A$ with arbitrarily preassigned minimal uniform index $k$.

In both examples we consider operators $A$ acting in the space $l^{1}$. If $x=\left(\xi_{1}, \xi_{2}, \cdots\right)$ and $y=\left(\eta_{1}, \eta_{2}, \cdots\right)$ are points of $l^{1}$ with $y=A x$, we take the defining equations to be

$$
\eta_{i}=\sum_{j=1}^{\infty} \alpha_{i j} \xi_{j}
$$$$
i=1,2, \cdots \text {. }
$$

Thus $A$ is represented by an infinite matrix. For operators acting in $l^{1}$ the norm is

$$
\|A\|=\sup _{j} \sum_{i=1}^{\infty}\left|\alpha_{i j}\right|
$$

EXAMPLE 1

For our first example we take the matrix representation of $A$ to have the form

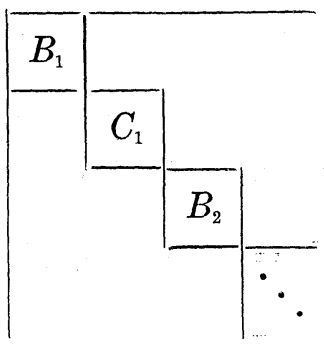

where the only nonzero matrix entries are in the square blocks $B_{1}, C_{1}, B_{2}, C_{2}, B_{3}, \cdots$ down the main diagonal. The block $B_{n}$ is to be a two-by-two block

$$
B_{n}: \begin{array}{ll}
\lambda_{n} & 0 \\
\mu_{n} & \lambda_{n}
\end{array},
$$

and the block $C_{n}$ is to have $r_{n}$ rows and columns, with the only nonzero elements 1 's in the subdiagonal:

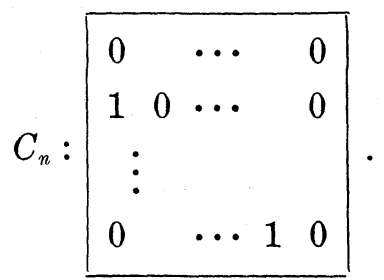

We require $0 \leqq r_{n}$, sup $r_{n}=r<\infty$. If $r_{n}=0$ the block $C_{n}$ is to be 
nonexistent, and if $r_{n}=1, C_{n}$ is to be a block with the single entry 0 . We assume $\left\{\lambda_{n}\right\}$ is a sequence of distinct nonzero numbers such that $\lambda_{n} \rightarrow 0 ;\left\{\mu_{n}\right\}$ can be any bounded sequence.

It is not difficult to compute $R_{\lambda}(A)$. Each point $\lambda_{n}$ is a pole of the second order and all other nonzero points are in $\rho(A)$. The block corresponding to $B_{n}$ in the matrix representing $R_{\lambda}(A)$ is

$$
\begin{array}{cc}
\hline \frac{1}{\lambda-\lambda_{n}} & 0 \\
\frac{\mu_{n}}{\left(\lambda-\lambda_{n}\right)^{2}} & \frac{1}{\lambda-\lambda_{n}} \\
\hline
\end{array}
$$

The block corresponding to $C_{n}$ is

$$
\begin{array}{cccc}
\hline \frac{1}{\lambda} & 0 & \cdots & 0 \\
\frac{1}{\lambda^{2}} & \frac{1}{\lambda} & & 0 \\
\vdots & & & 0 \\
\frac{1}{\lambda^{r_{n}}} & \cdots & \cdots & \frac{1}{\lambda} \\
\hline
\end{array}
$$

The matrices representing $E_{n}$ and $F_{n}$, respectively, have the blocks.

$$
\begin{array}{ll|}
1 & 0 \\
0 & 1
\end{array} \quad \begin{array}{ll}
0 & 0 \\
\mu_{n} & 0 \\
\hline
\end{array}
$$

in place of $B_{n}$, and all other entries are 0.

It is now easy to see that, if $l<m$,

$$
\|\left|\sum_{n=l}^{m}\left(\lambda_{n} E_{n}+F_{n}\right)^{p}\right| \mid=\sup _{l \leqq n \leqq m}\left[\left|\lambda_{n}\right|^{p}+p\left|\lambda_{n}^{p-1} \mu_{n}\right|\right] .
$$

Consequently, $A$ admits the uniform index 2 relative to $\left\{\lambda_{n}\right\}$. It admits the uniform index 1 if and only if $\mu_{n} \rightarrow 0$.

We can also see that

$$
\left\|\left(\lambda_{n} E_{n}+F_{n}\right)^{p}\right\|=\left|\lambda_{n}\right|^{p}+p\left|\lambda_{n}^{p-1} \mu_{n}\right| .
$$

Therefore $A$ admits the absolute index $p$ if and only if 


$$
\sum_{n=1}^{\infty}\left[\left|\lambda_{n}\right|^{p}+p\left|\lambda_{n}^{p-1} \mu_{n}\right|\right]<\infty .
$$

From this discussion we see that we can arrange for $A$ to have any prescribed minimal absolute index (greater than or equal to the minimal uniform index relative to $\left\{\lambda_{n}\right\}$ ). Or, we can also arrange for $A$ not to admit any absolute index at all. For instance, if $\lambda_{n}=[\log (n+1)]^{-1}$, and $\mu_{n}=n^{-1}$, the minimal uniform index is 1 and there is no absolute index. If $k>1, \lambda_{n}=n^{-k /(k-1)^{2}}$, and $\mu_{n}=1$, the minimal uniform index is 2 and the minimal absolute index is $k$.

Let us return to the general case of this example. Suppose we have the situation where the minimal uniform index is 2 . The operator $A$ is not canonical, but we can express it in the form $A=B+C$, where $B$ satisfies (63) and (64) with $p=2$, and $C$ is nilpotent (of order $r$ ), with $B C=C B=0$. We can define $B$ as the operator whose matrix representation is like the matrix representation of $A$ except that the blocks $C_{1}, C_{2}, \cdots$ have all been replaced by blocks of zeros. It can also be seen that

for each $x$, so that

$$
B x=\sum_{n=1}^{\infty}\left(\lambda_{n} E_{n}+F_{n}\right) x
$$

$$
B=\sum_{n=1}^{\infty}\left(\lambda_{n} E_{n}+F_{n}\right),
$$

with convergence in the strong (instead of the uniform) operator topology.

\section{EXAMPLE 2}

For this example we again use the matrix representation of $A$ and a scheme of blocks:

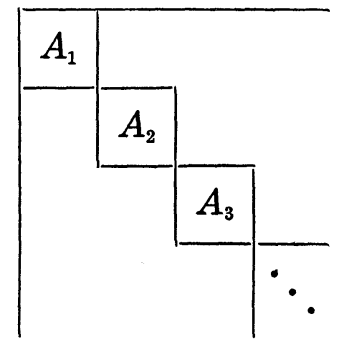

The form of the block $A_{n}$ is to be

$$
A_{n}: \begin{array}{ll}
\lambda_{2 n-1} & 0 \\
\mu_{n} & \lambda_{2 n}
\end{array} .
$$

We assume that $\left\{\lambda_{n}\right\}$ is a sequence of distinct nonzero numbers such 
that $\lambda_{n} \rightarrow 0$. For $\left\{\mu_{n}\right\}$ we take any bounded sequence. This time all the poles are simple; the block corresponding to $A_{n}$ in the matrix representation of $R_{\lambda}(A)$ is

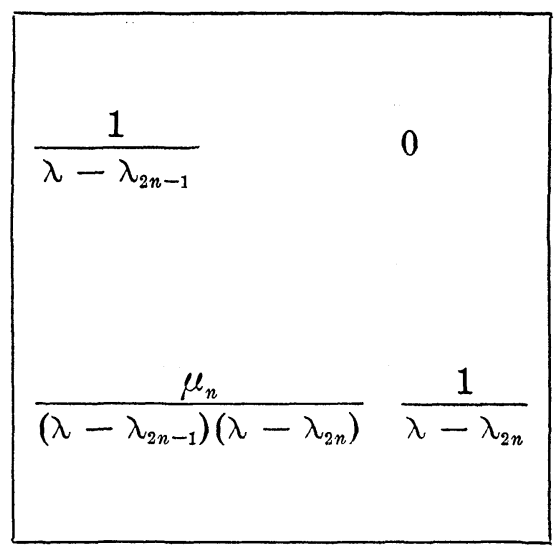

The matrix representing $E_{2 n-1}$ has just one nonzero block; it is in the position occupied by $A_{n}$, and it is

\begin{tabular}{|ll|}
\hline 1 & 0 \\
$\alpha_{n}$ & 0
\end{tabular},

where

$$
\alpha_{n}=\frac{\mu_{n}}{\lambda_{2 n-1}-\lambda_{2 n}} .
$$

The matrix representing $E_{2 n}$ is of the same type, but its sole nonzero block is

$$
\begin{array}{|ll|}
\hline 0 & 0 \\
-\alpha_{n} & 1 \\
\hline
\end{array}
$$

From these facts we can readily see that $A$ admits the absolute index $p$ if and only if

$$
\sum_{n=1}^{\infty}\left|\lambda_{2 n-1}\right|^{p}\left(1+\left|\alpha_{n}\right|\right)<\infty
$$

and

$$
\sum_{n=1}^{\infty}\left|\lambda_{2 n}\right|^{p} \beta_{n}<\infty,
$$

where $\beta_{n}=\max \left(1,\left|\alpha_{n}\right|\right)$. Equivalently, the condition is that each of the following four series must converge:

$$
\sum_{n=1}^{\infty}\left|\lambda_{2 n-1}\right|^{p}, \quad \sum_{n=1}^{\infty}\left|\lambda_{2 n}\right|^{p}, \quad \sum_{n=1}^{\infty}\left|\lambda_{2 n-1}\right|^{p}\left|\alpha_{n}\right|, \quad \sum_{n=1}^{\infty}\left|\lambda_{2 n}\right|^{p}\left|\alpha_{n}\right| .
$$


In investigating the question of a uniform index for $A$ relative to $\left\{\lambda_{i}\right\}$, it is convenient to begin by calculating $A^{p}$. The matrix representation of $A^{p}$ has the same form as that of $A$, with the block $A_{n}$ replaced by

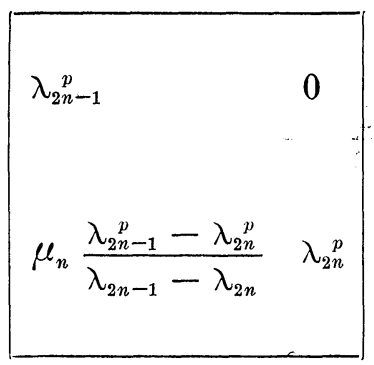

This block is the same as the sole nonzero block in the matrix representation of

$$
\lambda_{2 n-1}^{p} E_{2 n-1}+\lambda_{2 n}^{p} E_{2 n} .
$$

It is then easy to see that the matrix representation of

$$
A^{p}-\sum_{k=1}^{2 n} \lambda_{k}^{p} E_{k}
$$

is derived from the matrix representation of $A^{p}$ simply by putting blocks of zeros in place of the first $n$ blocks down the diagonal. From this it may be proved that $A$ admits the uniform index $p$ relative to $\left\{\lambda_{n}\right\}$ if and only if

$$
A^{p}=\sum_{n=1}^{\infty} \lambda_{n}^{p} E_{n}
$$

and that this occurs if and only if

$$
\left\|\lambda_{2 n-1}^{p} E_{2 n-1}+\lambda_{2 n}^{p} E_{2 n}\right\| \rightarrow 0
$$

and

$$
\left\|\lambda_{2 n-1}^{p} E_{2 n-1}\right\| \rightarrow 0
$$

as $n \rightarrow \infty$.

Condition (67) is equivalent to

$$
\max \left\{\left|\lambda_{2 n-1}\right|^{p}+\left|\lambda_{2 n-1}^{p}-\lambda_{2 n}^{p}\right|\left|\alpha_{n}\right|,\left|\lambda_{2 n}\right|^{p}\right\} \rightarrow 0,
$$

and condition (68) is equivalent to

$$
\left|\lambda_{2 n-1}\right|^{p}\left(1+\left|\alpha_{n}\right|\right) \rightarrow 0 \text {. }
$$

Since. we assumed at the outset that $\lambda_{n} \rightarrow 0$, it is now easy to see that $A$ admits the uniform index $p$ relative to $\left\{\lambda_{n}\right\}$ if and only if

$$
\frac{\left.\left|\lambda_{2 n-1}\right|\right|^{p}\left|\mu_{n}\right|}{\left|\lambda_{2 n-1}-\lambda_{2 n}\right|} \rightarrow 0 \text { and } \frac{\left|\lambda_{2 n}\right|^{p}\left|\mu_{n}\right|}{\left|\lambda_{2 n-1}-\lambda_{2 n}\right|} \rightarrow 0
$$


If we take $\lambda_{n}=n^{-1}$, it turns out that $A$ admits the uniform index $p$ relative to $\left\{\lambda_{n}\right\}$ if and only if $\mu_{n} n^{2-n} \rightarrow 0$, and $A$ admits the absolute index $p$ if and only if

$$
\sum_{n=1}^{\infty} \frac{\left|\mu_{n}\right|}{n^{p-2}}<\infty
$$

In particular, since $\left\{\mu_{n}\right\}$ is bounded, $A$ certainly admits the uniform index 3 and the absolute index 4 , but these indices are not necessarily minimal.

If we take $\lambda_{n}=n^{-1 / k}$ (with $k$ a positive integer) a simple calculation with the binomial series shows that each of the expressions

$$
\frac{\left|\lambda_{2 n-1}\right|^{p}}{\left|\lambda_{2 n-1}-\lambda_{2 n}\right|} \text { and } \frac{\left|\lambda_{2_{n}}\right|^{p}}{\left|\lambda_{2 n-1}-\lambda_{2 n}\right|}
$$

is asymptotically equivalent to

$$
k\left(\frac{1}{2 n-1}\right)^{(p-1-k) / k}
$$

Consequently, $A$ admits the uniform index $p$ relative to $\left\{\lambda_{n}\right\}$ if and only if

$$
n^{(k+1-p) / k} \quad \mu_{n} \rightarrow 0 .
$$

With $\mu_{n}=n^{-2 / k}$, we can see that $A$ has minimal uniform index $k$ relative to $\left\{\lambda_{n}\right\}$. These same choices of $\lambda_{n}$ and $\mu_{n}$ give $A$ the minimal absolute index $2 k$.

\section{REFERENCES}

1. Earl Berkson, Sequel to a paper of A. E. Taylor, Pacific J. Math., 10 (1960), 767-776.

2. John Derr, Multiple poles of the resolvent for operators of finite Mittag-Leffler type, Dostoral dissertation, University of California, Los Angeles, 1962.

3. E. Hille and R. S. Phillips, Functional analysis and semi-groups (revised edition), American Mathematical Society Colloquium Publications, vol. 31, New York, 1957.

4. J. D. Newburgh, The variation of spectra, Duke Math. J., 18 (1951), 165-176.

5. Angus E. Taylor, Introduction to functional analysis, John Wiley, New York, 1958.

6. - Mittag-Leffler expansions and spectral theory, Pacific J. Math., 10 (1960), 1049-1066.

7. - Spectral theory and Mittag-Leffler type expansions of the resolvent, Proceedings of the International Symposium on Linear Spaces, The Hebrew University, Jerusalem, 1960.

THE RAND CORPORATION

The University of California, Los Angeles 



\section{PACIFIC JOURNAL OF MATHEMATICS}

\section{EDITORS}

Ralph S. Phillips

Stanford University

Stanford, California

M. G. Arsove

University of Washington

Seattle 5, Washington
A. L. Whiteman

University of Southern Californla

Los Angeles 7, California

Lowell J. Paige

University of California

Los Angeles 24, California

\section{ASSOCIATE EDITORS}

E. F. BECKENBACH

D. DERRY

H. L. ROYDEN

E. G. STRAUS

T. M. CHERRY

M. OHTSUKA

E. SPANIER

F. WOLF

\section{SUPPORTING INSTITUTIONS}

UNIVERSITY OF BRITISH COLUMBIA

STANFORD UNIVERSITY

CALIFORNIA INSTITUTE OF TECHNOLOGY

UNIVERSITY OF CALIFORNIA

MONTANA STATE UNIVERSITY

UNIVERSITY OF TOKYO

UNIVERSITY OF UTAH

UNIVERSITY OF NEVADA

NEW MEXICO STATE UNIVERSITY

OREGON STATE UNIVERSITY

UNIVERSITY OF OREGON

OSAKA UNIVERSITY

WASHINGTON STATE UNIVERSITY

UNIVERSITY OF WASHINGTON

UNIVERSITY OF SOUTHERN CALIFORNIA

AMERICAN MATHEMATICAL SOCIETY CALIFORNIA RESEARCH CORPORATION SPACE TECHNOLOGY LABORATORIES NAVAL ORDNANCE TEST STATION 


\section{Pacific Journal of Mathematics}

\section{Vol. 12, No. $1 \quad$ January, 1962}

Jonathan L. Alperin, Groups with finitely many automorphisms $\ldots \ldots \ldots \ldots \ldots \ldots \ldots \ldots$

Martin Arthur Arkowitz, The generalized Whitehead product ................ 7

John D. Baum, Instability and asymptoticity in toplogical dynamics . . . . . . . . . . 25

William Aaron Beyer, Hausdorff dimension of level sets of some Rademacher series .... $\quad 35$

Frank Herbert Brownell, III, A note on Cook's wave-matrix theorem . . . . . . . . . . . . . 47

Gulbank D. Chakerian, An inequality for closed space curves ................. 53

Inge Futtrup Christensen, Some further extensions of a theorem of Marcinkiewicz ....... 59

Charles Vernon Coffman, Linear differential equations on cones in Banach spaces . . . . . 69

Eckford Cohen, Arithmetical notes. III. Certain equally distributed sets of integers . . . . . 77

John Irving Derr and Angus E. Taylor, Operators of meromorphic type with multiple poles

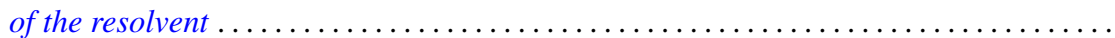

Jacob Feldman, On measurability of stochastic processes in products space .............

Robert S. Freeman, Closed extensions of the Laplace operator determined by a general class of boundary conditions, for unbounded regions ......................

Robert E. Fullerton, Geometric structure of absolute basis systems in a linear topological

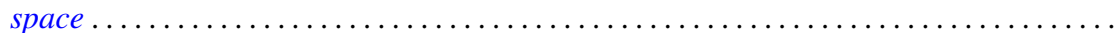

Dieter Gaier, On conformal mapping of nearly circular regions

Andrew Mattei Gleason and Hassler Whitney, The extension of linear functionals defined

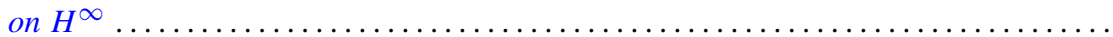

Seymour Goldberg, Closed linear operators and associated continuous linear

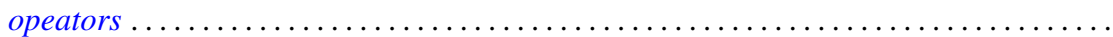

Basil Gordon, Aviezri Siegmund Fraenkel and Ernst Gabor Straus, On the determination of sets by the sets of sums of a certain order

Branko Grünbaum, The dimension of intersections of convex sets. .

Paul Daniel Hill, On the number of pure subgroups

Robert Peter Holten, Generalized Goursat problem . .

Alfred Horn, Eigenvalues of sums of Hermitian matrices ...........

Henry C. Howard, Oscillation and nonoscillation criteria for

$$
y^{\prime \prime}(x)+f(y(x)) p(x)=0
$$

Taqdir Husain, $S$-spaces and the open mapping theorem ...

Richard Eugene Isaac, Markov processes and unique stationary probability measures ...

John Rolfe Isbell, Supercomplete spaces ....................

John Rolfe Isbell, On finite-dimensional uniform spaces. II .........

N. Jacobson, A note on automorphisms of Lie algebras ..............

Antoni A. Kosinski, A theorem on families of acyclic sets and its applications

Marvin David Marcus and H. Minc, The invariance of symmetric functions of singular values...

Ralph David McWilliams, A note on weak sequential convergence.

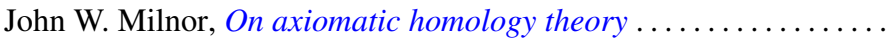

Victor Julius Mizel and Malempati Madhusudana Rao, Nonsymmetric projections in

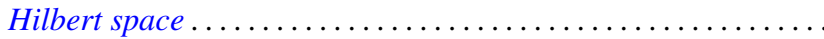

Calvin Cooper Moore, On the Frobenius reciprocity theorem for locally compact

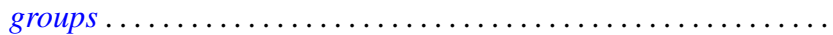

Donald J. Newman, The Gibbs phenomenon for Hausdorff means . 